Electronic Supplementary Information (ESI) for

\title{
Long-Term Dynamic Imaging of Cellular Processes Using an AIE Lipid Order Probe in the Dual-Color Mode
}

Yue Zheng ${ }^{1}$, Yiwen Ding ${ }^{1}$, Xiaokun Zheng ${ }^{1}$, Chu Zhang ${ }^{1}$, Yanli Zhang ${ }^{2}$, Yu Xiang ${ }^{1}$ and Aijun Tong ${ }^{1 *}$

1. Department of Chemistry, Beijing Key Laboratory for Microanalytical Methods and Instrumentation, Key

Laboratory of Bioorganic Phosphorus Chemistry and Chemical Biology (Ministry of Education), Tsinghua University, Beijing 100084 (P. R. China)

2. Imaging Core Facility, Technology Center for Protein Science, Tsinghua University, Beijing 100084 (P. R. China)

Email: tongaj@mail.tsinghua.edu.cn 


\section{Table of Contents}

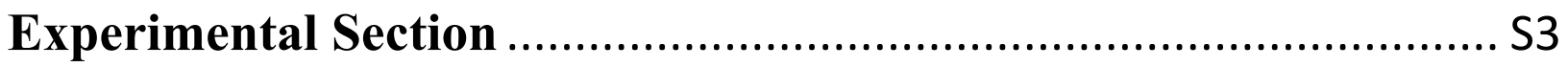

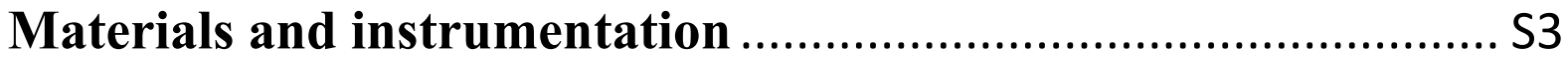

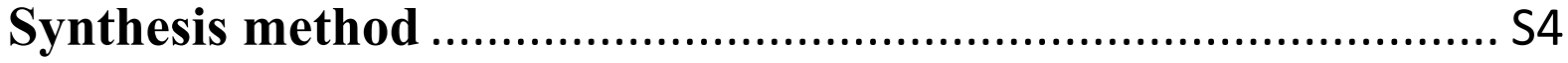

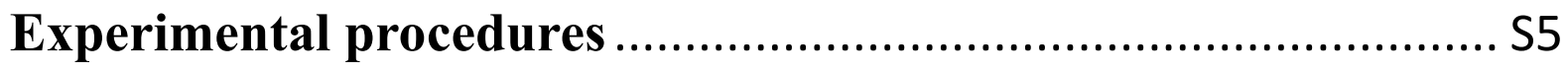

Additional Figures and Tables...................................................... S8

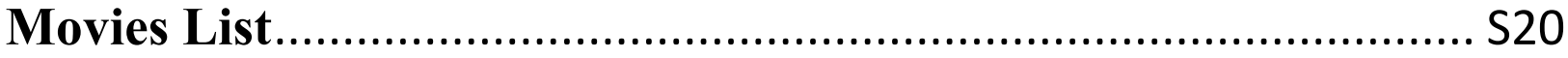




\section{Experimental Section}

\section{Materials and instrumentation}

1-Bromopentadecane (\#B0936, purity 98\%), 3-bromo-N,N,N-trimethylpropan-1-aminium bromide (\#B802775, purity 98\%), boron tribromide (\#C19890, 1 M solution in methylene chloride) were purchased from J\&K (Beijing, China). 4-[Bis(4-methoxyphenyl)amino]benzaldehyde (\#B4905, purity 98\%), 4methylpyridine (\#P0417, purity 98\% ) were purchased from TCI (Shanghai, China). Piperidine (\#411027, purity 99.5\%), 1,2-Dioleoyl-sn-glycero-3-phosphocholine (DOPC, \#P6354, purity 97\%), N-acyl-4sphingenyl-1-O-phosphorylcholine (SM, \#85615, purity $\geq 98.0 \%$ ), cholesterol (Chol, \#C8667, purity $\geq 99 \%$ ), sucrose (\#V900116, purity 99\%), D-glucose (\#G8270, purity $\geq 99.5 \%$ ), erastin (\#E7781, purity $\geq 98 \%$ ) and indium tin oxide (ITO) glass plate (\#703176) were purchased from Sigma-Aldrich Co., Ltd. (Shanghai, China). All chemicals and materials were used as received without further purification or treatment. Analytical purity organic solvents and inorganic salts were purchased from Sinopharm Chemical Reagent Beijing Co. (Beijing, China). All aqueous solutions were prepared using ultrapure water, which was obtained through a Millipore Milli-Q water purification system (Billerica, MA) and had an electric resistance at 18.2 M $\Omega$. HeLa cells were purchased from National Platform of Experimental Cell Resources for Sci-Tech (Beijing, China). Dulbecco's modified Eagle's medium (DMEM), fetal bovine serum (FBS), Dulbecco's Phosphate Buffered Saline (DPBS, 1×), penicillin-streptomycin and 0.25\% Trypsin were purchased from Corning Cellgro (NY, USA). Cell Counting Kit-8 (CCK-8 assay for cell viability) was purchased from Dojindo Molecular Technologies, Inc. (Tokyo, Japan). 3,3'Dioctadecyloxacarbocyanine Perchlorate (DiO, \#D275) was purchased from Invitrogen Trading Co., Ltd. (Shanghai, China).

NMR spectra were recorded using a JOEL JNM-ECA400 spectrometer (Tokyo, Japan) operated at $400 \mathrm{MHz}$. Mass spectra were obtained on a Shimadzu (Kyoto, Japan) MS-IT-TOF ion trap time-of-flight mass spectrometry. The fluorescent photographs were taken by a Canon EOS 600D camera. All fluorescence spectra were recorded with a JASCO FP-8600 fluorescence spectrometer (Tokyo, Japan). Dynamic light scattering for nanoparticle sizes were determined at $25^{\circ} \mathrm{C}$ by a Horiba's nanoparticle analyzer Nano SZ-100 (Kyoto, Japan). 
Giant unilamellar vesicles (GUVs) fluorescence imaging, photostability testing and live cell imaging for osmotic shock were performed on Olympus FV 1200 confocal laser scanning microscope (CLSM, Tokyo, Japan) using 60X lens.

For fluorescence lifetime imaging (FLIM-FCS), time-correlated single-photon counting (TCSPC) data sets were acquired on an inverted Olympus FV1200 microscope with a 60X NA equipped with a PicoQuant picoHarp300 (Berlin, Germany) controller. The nondescanned emission was collected from a PicoQuant bandpass filter and detected by a PicoQuant MPD SPAD detector. Photon data were analyzed using SymPhoTime64 mage software.

2D and 3D colocalization of as well as real-time dynamically apoptosis and ferroptosis imaging were operated using a Dragonfly200 Andor Spinning Disk Confocal Microscope in living cell station (containing $5 \% \mathrm{CO}_{2}$ with appropriate humidity at $37^{\circ} \mathrm{C}$ ) with filter $521 \pm 38(405 \mathrm{~nm}$ laser) and $594 \pm 40$ (488 nm laser) using $60 \mathrm{X}$ or $40 \mathrm{X}$ lens. GUV fluorescence intensity statistics, colocalization and live cell dynamically imaging results were all analyzed using Imaris 9.5 software.

\section{Synthesis}

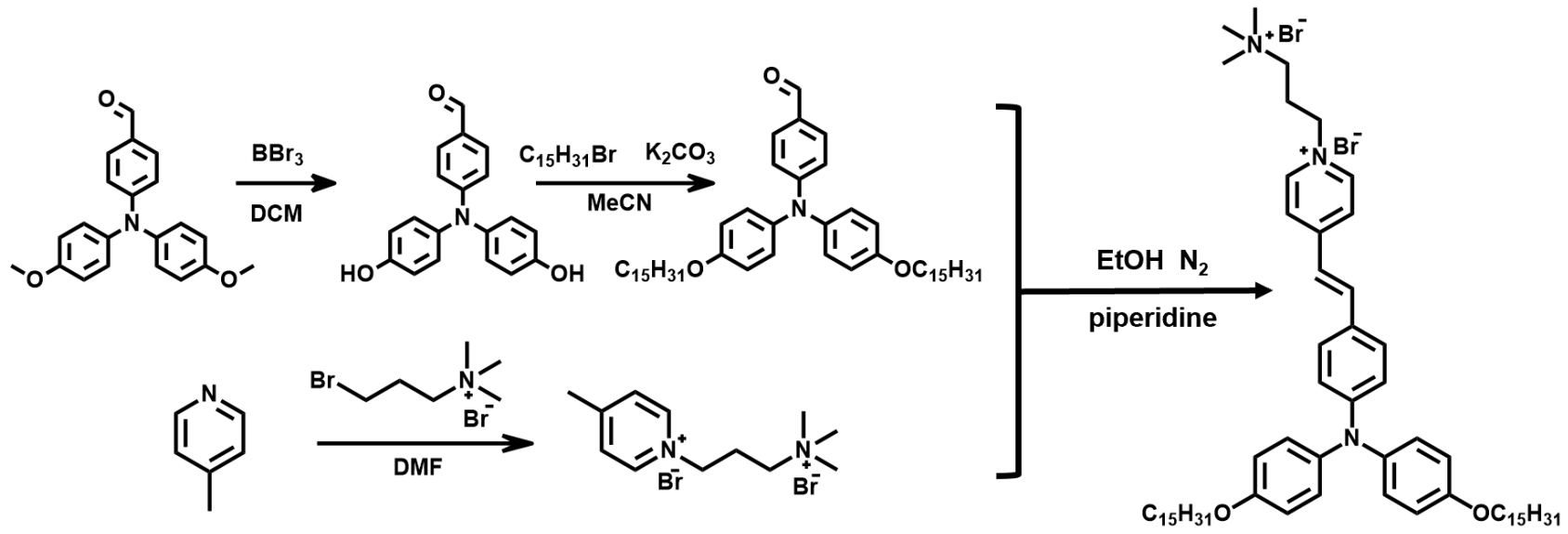

Scheme S1 Synthetic route of TPNPDA-C15

TPNPDA-C15 was synthesized following the reported method in our previous work ${ }^{1}$ to get dark red powder with yield as 85.20\%. Synthetic route shows in Scheme S1. ${ }^{1} \mathrm{H}$ NMR (methanol-d3, $400 \mathrm{MHz}$, TMS), $\delta(\mathrm{ppm}): 0.86(\mathrm{t}, \mathrm{J}=8.0 \mathrm{~Hz}, 6 \mathrm{H}), 1.24(\mathrm{~m}, 48 \mathrm{H}), 1.43(\mathrm{~m}, 2 \mathrm{H}), 1.73(\mathrm{~m}, 4 \mathrm{H}), 2.49(\mathrm{t}, \mathrm{J}=8.0 \mathrm{~Hz}, 2 \mathrm{H})$, $3.16(\mathrm{~s}, 9 \mathrm{H}), 3.93$ (t, J=8.0 Hz, 4H), 4.52 (t, J=8.0 Hz, 2H), 6.77-6.80 (m, 2H), 6.87-6.89 (m, 4H), 7.05- 
7.07 (m, 4H), $7.16(\mathrm{~d}, \mathrm{~J}=16.0 \mathrm{~Hz}, 1 \mathrm{H}), 7.49-7.51(\mathrm{~m}, 2 \mathrm{H}), 7.84(\mathrm{~d}, \mathrm{~J}=16.0 \mathrm{~Hz}, 1 \mathrm{H}), 8.04-8.06(\mathrm{~m}, 2 \mathrm{H})$, 8.66-8.68 (m, 2H). ${ }^{13} \mathrm{C}$ NMR (Tetrahydrofuran - d8 : $\left.\mathrm{D}_{2} \mathrm{O}=1: 1(\mathrm{v} / \mathrm{v}), 101 \mathrm{MHz}\right), \delta(\mathrm{ppm}): 13.68,22.68$, $23.41,24.81,26.17,29.40,29.50,29.71,29.74,31.93,53.32,56.82,62.65,67.90,115.25,118.40,119.85$, 123.71, 127.30, 129.78, 139.65, 141.04, 144.06, 150.56, 154.28, 156.41, 180.49. MS (m/z): calcd. for $\mathrm{C}_{61} \mathrm{H}_{95} \mathrm{O}_{2} \mathrm{~N}_{3} \mathrm{Br}_{2}[\mathrm{M}-2 \mathrm{Br}]^{2+}: 450.8707$, found: 450.8692 .

\section{Experimental procedures}

\section{Analytical procedures}

Weighed $10.6 \mathrm{mg}$ TPNPDA-C15 in a glass vial and then added $1 \mathrm{~mL}$ ethanol to dissolve it, ultrasonic for $10 \mathrm{~min}$ to prepare $10 \mathrm{mM}$ TPNPDA-C15 stock solution. In a typical detection, $10 \mu \mathrm{L}$ of stock solutions were added into a test tube, which was diluted to other solvents to get solutions with estimated concentrations and the fluorescence spectra were recorded. Dynamic light scattering analysis was taken using the same samples.

\section{Cell culture}

Hela cells were cultured in the DMEM containing $10 \%$ FBS and antibiotics $(100 \mathrm{units} / \mathrm{mL}$ penicillin and $0.1 \mathrm{mg} / \mathrm{mL}$ streptomycin) in a $5 \% \mathrm{CO}_{2}$ humidity incubator at $37^{\circ} \mathrm{C}$. For confocal imaging, cells were grown in a $35 \mathrm{~mm}$ Petri dish with a coverslip at $37{ }^{\circ} \mathrm{C}$ overnight after attachment.

\section{Cytotoxicity}

CCK-8 assays were used to evaluate the cytotoxicity of the presented probes.

Cells were incubated in $96-w e l l$ plates $(100 \mu \mathrm{L}$ per well) at a density of $6000-8000$ cells/well. Culture medium without cells was also introduced into the wells (100 $\mu \mathrm{L}$ per well) as blank. Stock solutions of TPNPDA-C15 in ethanol with different concentrations were prepared. After overnight culturing, $1 \mu \mathrm{L}$ stock solutions or ethanol were added to each well to get final dye concentrations at $0 \mu \mathrm{M}, 5 \mu \mathrm{M}, 10 \mu \mathrm{M}$, $15 \mu \mathrm{M}$ and $20 \mu \mathrm{M}$. And 24 hours later, $10 \mu \mathrm{L}$ CCK-8 solution was added into each well and cells were incubated for further $2 \mathrm{~h}$. The absorption of each well at $450 \mathrm{~nm}$ was recorded via a Molecular Device SpectraMax M3 plate reader, marked as As (for wells containing cell, culture medium and probes), Ac (for wells containing cell and culture medium), Ab1 (for wells containing culture medium and probes), Ab2 (for wells containing only culture medium). Each trial was performed with 5 wells parallel. The 
viability of cells was then calculated with the following equation:

$$
\text { Cell Viability }=\frac{A s-A b 1}{A c-A b 2}
$$

\section{Co-localization experiments}

$1 \mathrm{mM}$ DiO stock solution was prepared in DMSO. After that, $5 \mu \mathrm{L}$ of DiO stock solution and $10 \mu \mathrm{L}$ of TPNPDA-C15 stock solution were added in $1 \mathrm{~mL}$ being cultured HeLa cells. The cells were incubated with the probes at $37^{\circ} \mathrm{C}$. After $10 \mathrm{~min}$, washed cells for 3 times by $1 \times$ DPBS buffer, then added $1 \mathrm{~mL}$ culture medium for cell imaging. Fluorescent signal of DiO was excited by $488 \mathrm{~nm}$ laser and collected using $521 \pm 38 \mathrm{~nm}$ filter and TPNPDA-C15 was collected using $594 \pm 40 \mathrm{~nm}$ filter. Confocal imaging scans are performed every $1 \mu \mathrm{m}$ interval on the $\mathrm{z}$ axis. The attached single layer $2 \mathrm{D}$ confocal imaging and the 3D stereo fluorescence imaging of Hela cells were obtained for colocalization analysis, respectively.

\section{Fluorescence lifetime imaging}

HeLa cells stained by TPNPDA-C15 were used for FLIM analysis before and after sucrose solution added. After imaging on CLSM, samples were then excited by the picosecond $405 \mathrm{~nm}$ or $488 \mathrm{~nm}$ pulses generated by a $0.2 \mathrm{MHz}$ laser. The emission signals were collected though a 550/49 nm or 615/60 nm bandpass filter, respectively. Images of $512 \times 512$ pixels were obtained. Analysis of the acquired FLIM data was performed by first binning ( 3 pixels $\times 3$ pixels) the time dependent photon image and assigning a minimum threshold count of 100 recorded photons for modeling. Lifetime values derived from the exponential fits are then displayed as a heat-map image.

\section{Photocytotoxicity}

The photocytotoxicity of TPNPDA-C15 was also evaluated using CCK-8 assays. The same cell samples as cytotoxicity were prepared. For photocytotoxicity detection, cells were incubated with different concentrations of TPNPDA-C15 for 10 min and then with or without a Thorlabs DC4104 (NJ, USA) LED laser ( $455 \mathrm{~nm} 310 \mathrm{~mW}$ ) irradiation for $10 \mathrm{~min}$. Later, $10 \mu \mathrm{L} \mathrm{CCK}-8$ solution was added into each well and cells were incubated for further $2 \mathrm{~h}$. The absorption of each well at $450 \mathrm{~nm}$ was recorded via plate reader, marked as As (for wells containing cell, culture medium and probes), Ac (for wells containing cell and culture medium), Ab1 (for wells containing culture medium and probes), Ab2 (for wells containing only 
culture medium). Each trial was performed with 6 wells parallel. The viability of cells was then calculated with the following equation:

$$
\text { Cell Viability }=\frac{A s-A b 1}{A c-A b 2}
$$

\section{Photostability}

HeLa cells were fixed by and then stained with $10 \mu \mathrm{M}$ DiO or TPNPDA-C15 using the staining method mentioned above. For photostability testing, each cell samples were under $488 \mathrm{~nm}$ semiconductor laser $(52 \mu \mathrm{W})$ with 800 scans for a total of $2166 \mathrm{~s}$ ( $36 \mathrm{~min})$ on an Olympus FV 1200 confocal microscope. The emissions of DiO were collected at 500-600 nm and TPNPDA-C15 were collected at 600-700 nm. For photo-induced fluorescence wavelength switch testing, cell samples stained by TPNPDA-C15 were scanned by $405 \mathrm{~nm}$ and $488 \mathrm{~nm}$ semiconductor laser $(52 \mu \mathrm{W})$ for $36 \mathrm{~min}$. Yellow and red emissions were collected at 440-540 nm and 600-700nm, respectively. Quantization by line plots was accomplished by Olympus software of 800 confocal pictures. 6 fluorescent area on cell membranes were picked on each picture to calculate average fluorescence intensity as parallel. 


\section{Additional Figures and Tables}

\begin{tabular}{|c|c|c|c|}
\hline Solvent & Ethanol & $\begin{array}{c}99 \% \text { Ethyl } \\
\text { acetate/ethanol }\end{array}$ & $\begin{array}{c}99 \% \\
\text { Glycerin/ethanol }\end{array}$ \\
\hline $\begin{array}{c}\lambda_{\max } 1(\mathrm{~nm}) / \\
\varepsilon\left(10^{4} \mathrm{~L} \cdot \mathrm{mol}^{-1} \cdot \mathrm{cm}^{-1}\right)\end{array}$ & $282 / 0.6952$ & $299 / 1.3595$ & $299 / 1.6076$ \\
\hline $\begin{array}{c}\lambda_{\max } 2(\mathrm{~nm}) / \\
\varepsilon\left(10^{4} \mathrm{~L} \cdot \mathrm{mol}^{-1} \cdot \mathrm{cm}^{-1}\right)\end{array}$ & $510 / 1.6302$ & $471 / 2.3609$ & $501 / 1.0648$ \\
\hline$\lambda_{\text {ex }} / \lambda_{\mathrm{em}}(\mathrm{nm})$ & - & $\sim 373 / 567$ & $\sim 500 / 650$ \\
\hline$\tau(\mathrm{ns})$ & - & 2.3083 & 0.1128 \\
\hline Quantum yield $(\%)$ & - & 2.2 & 3.2 \\
\hline Average diameter $(\mathrm{nm})$ & 5.5 & 104.1 & 2.8 \\
\hline
\end{tabular}

Table S1 Fluorescence properties of TPNPDA analogs.

(a) $0,10,20,30,40,50,60,70,80,90,99$.

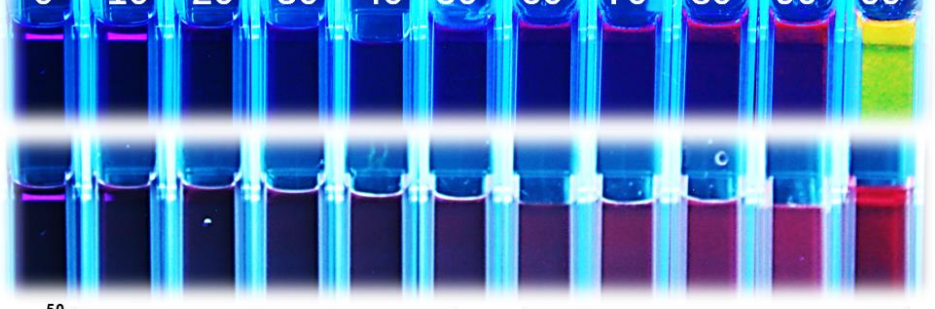

(b)
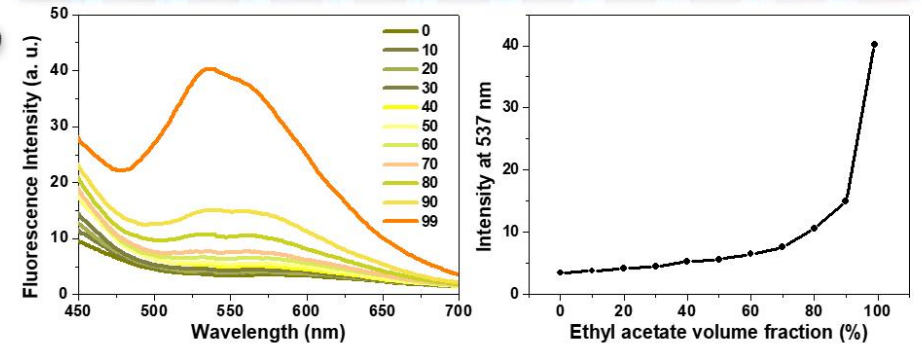

(c)
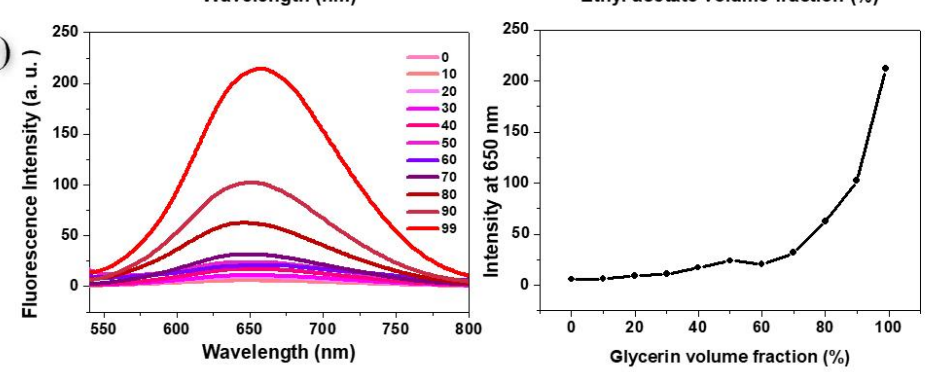

Figure S1 (a) Photographs of TPNPDA-C15 solution with different ethyl acetate (top) or glycerin (bottom) /ethanol volume fractions under $365 \mathrm{~nm}$ UV light. (b) Fluorescence spectra and intensity at $537 \mathrm{~nm}$ of 
TPNPDA-C15 with different ethyl acetate/ethanol volume fractions, $\lambda_{\mathrm{ex}}=373 \mathrm{~nm}$. (c) Fluorescence spectra and intensity at $650 \mathrm{~nm}$ of TPNPDA-C15 with different glycerin /ethanol volume fractions, $\lambda_{\mathrm{ex}}=500 \mathrm{~nm}$.

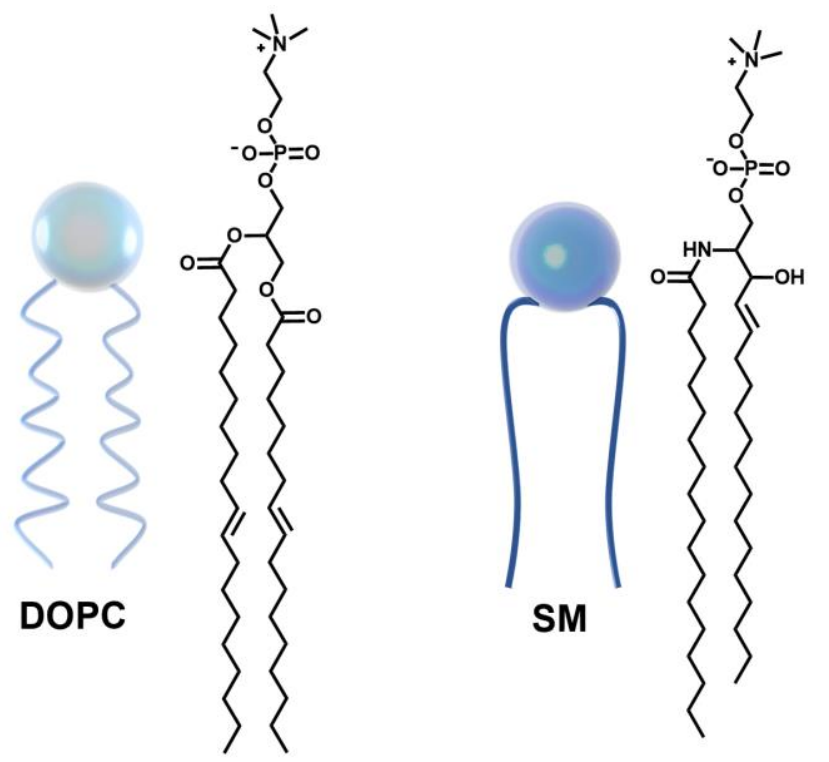

Figure S2 Chemical structures and the schematic drawings of DOPC and SM.

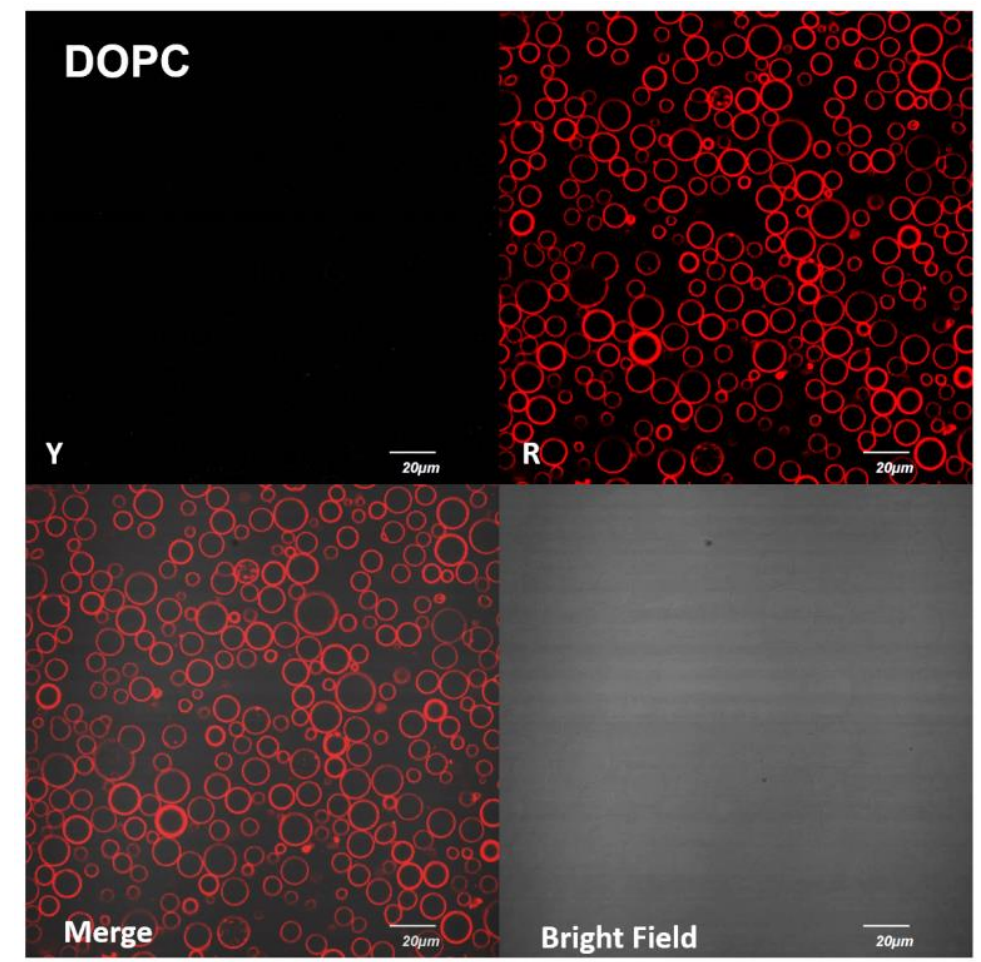

Figure S3 Confocal fluorescence images of DOPC GUVs stained with TPNPDA-C15. Scale bars are 20 $\mu \mathrm{m}$. 


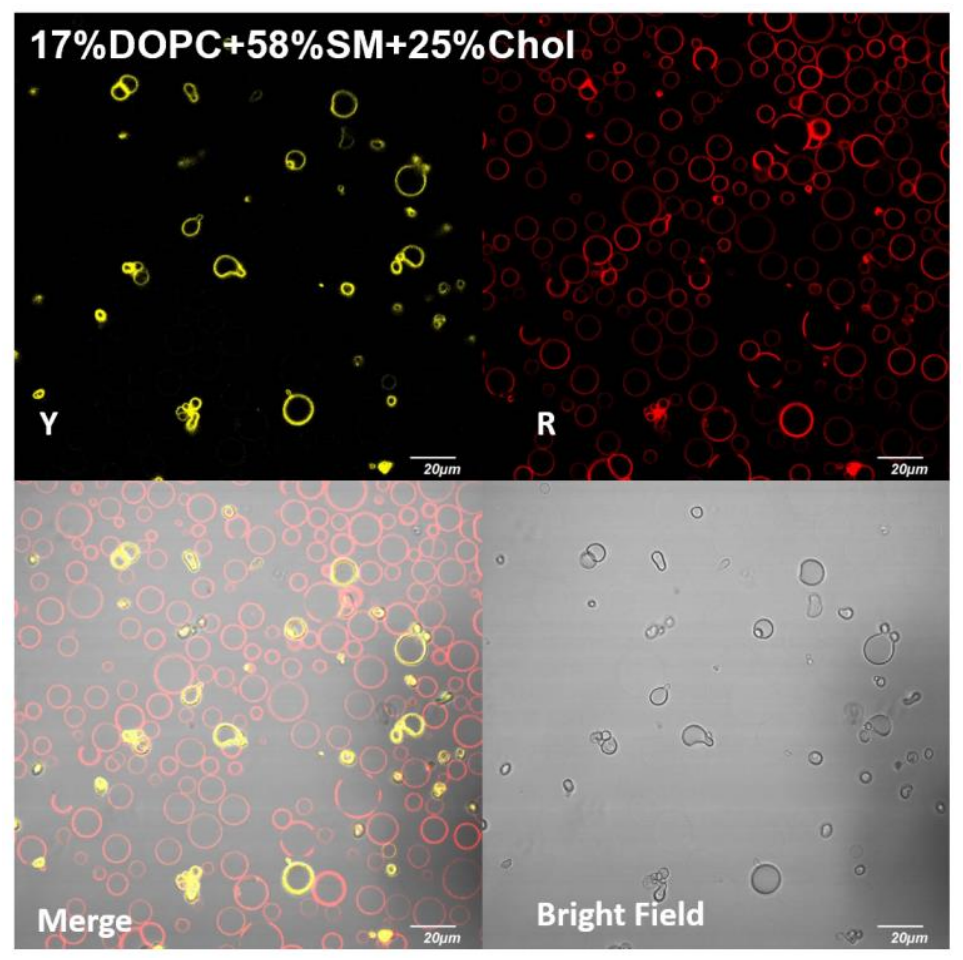

Figure S4 Confocal fluorescence images of 17\%DOPC $+58 \% \mathrm{SM}+25 \%$ Cholesterol GUVs stained with TPNPDA-C15. Scale bars are $20 \mu \mathrm{m}$.

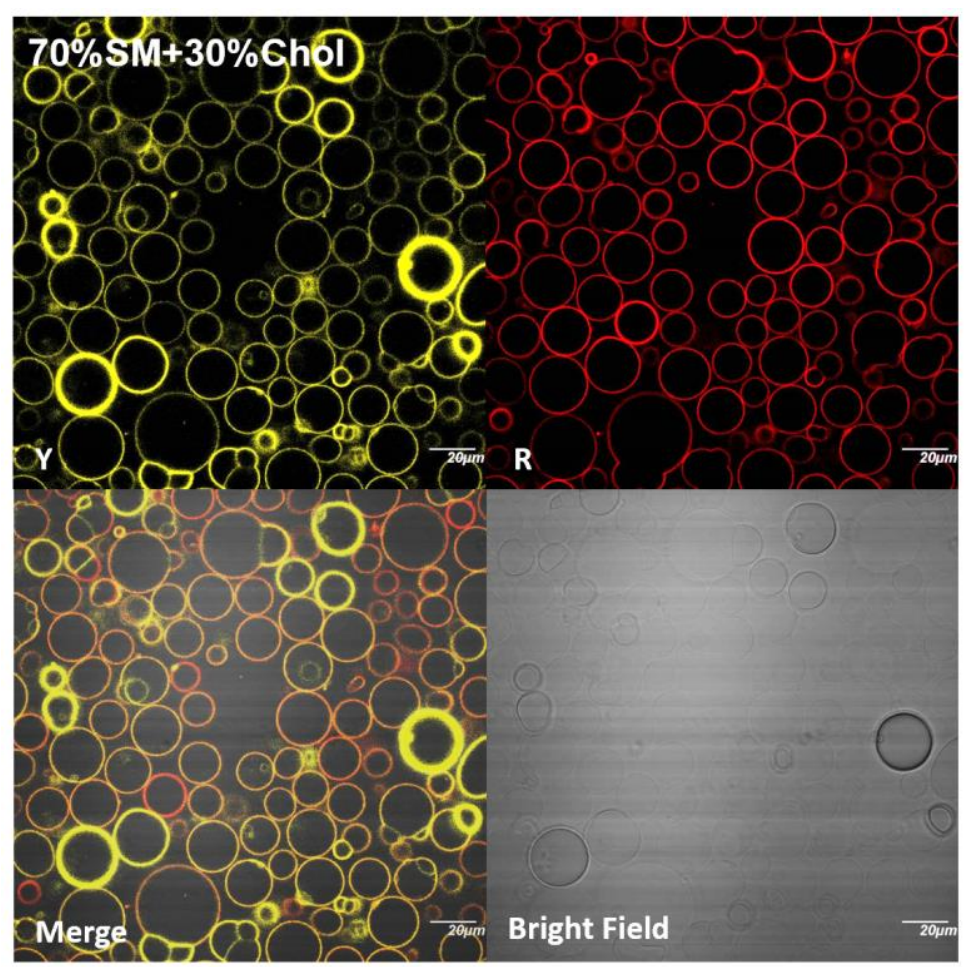

Figure S5 Confocal fluorescence images of 70\%SM + 30\%Cholesterol GUVs stained with TPNPDAC15. Scale bars are $20 \mu \mathrm{m}$. 


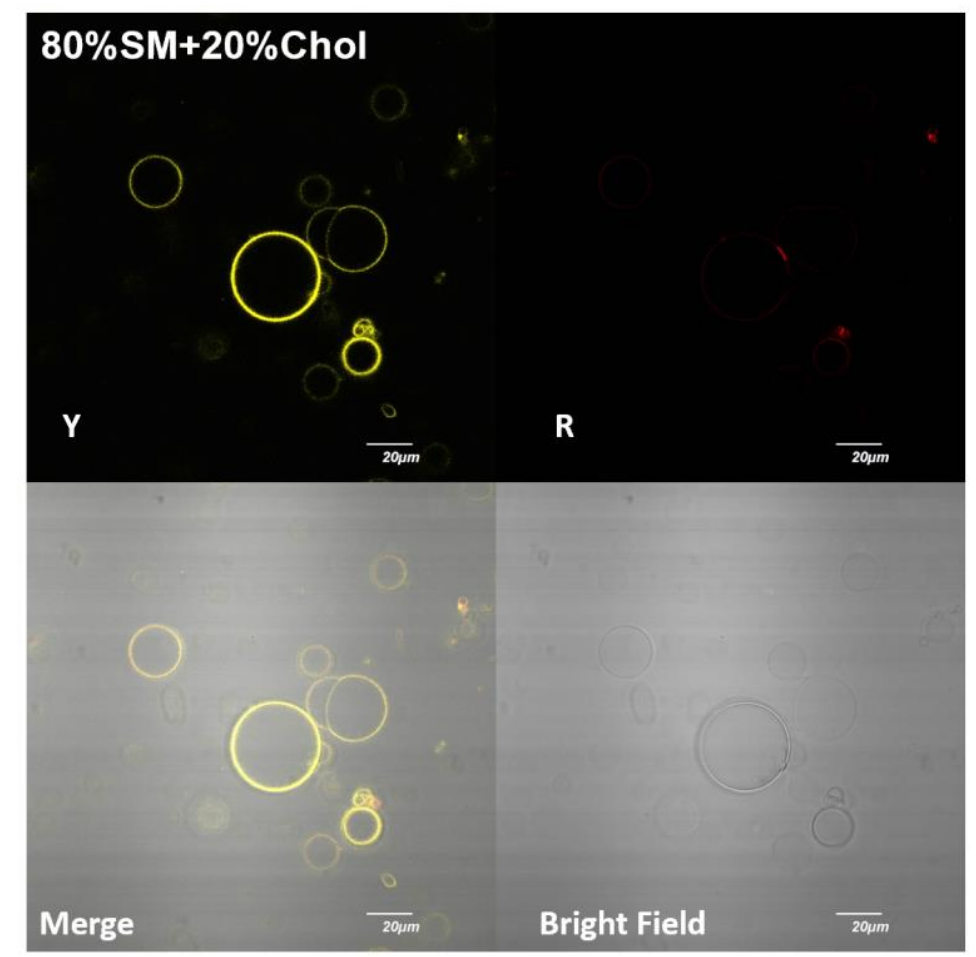

Figure S6 Confocal fluorescence images of $80 \% \mathrm{SM}+20 \%$ Cholesterol GUVs stained with TPNPDAC15. Scale bars are $20 \mu \mathrm{m}$.

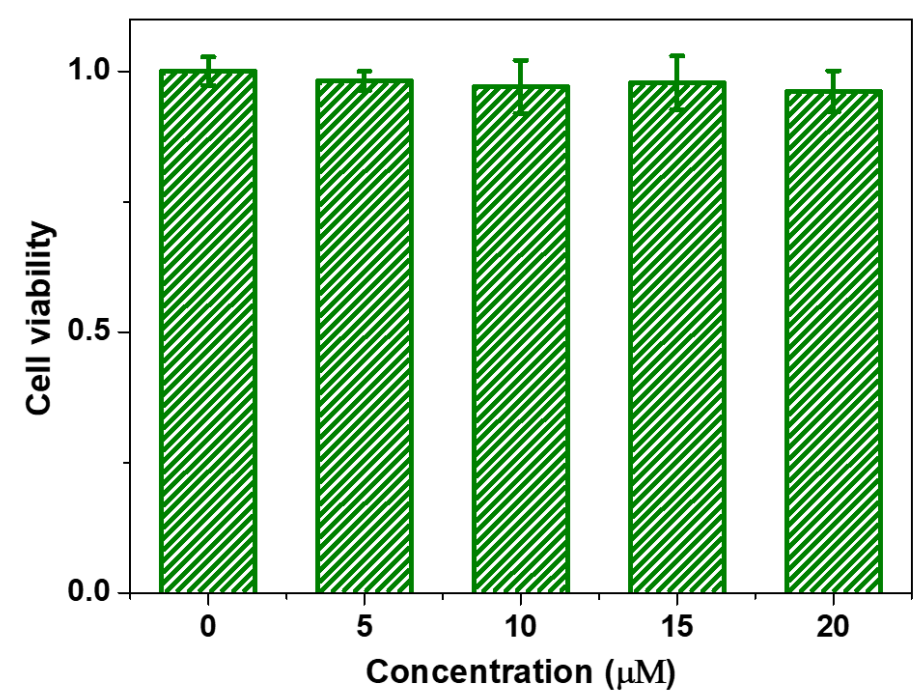

Figure S7 Cytotoxicity of TPNPDA-C15 for HeLa cells. Error bars represent standard error of mean for five repeated measurements. 

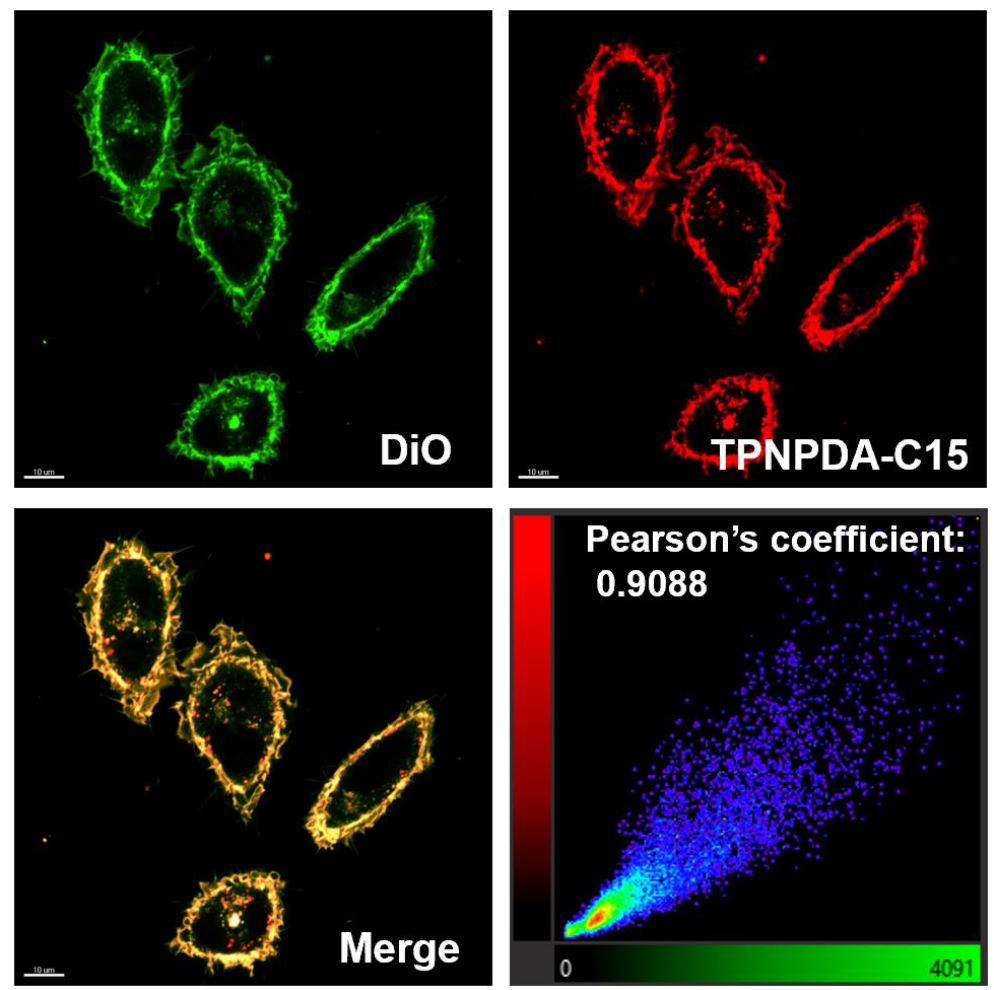

Figure S8 Confocal fluorescence images (single layer of the bottom of the cells) and co-localization scatter plot of HeLa cells stained with TPNPDA-C15 and DiO. Scale bars are $10 \mu \mathrm{m}$. Pearson's correlation coefficient is 0.91 .

\section{Osmotic pressure (П) / Osm}

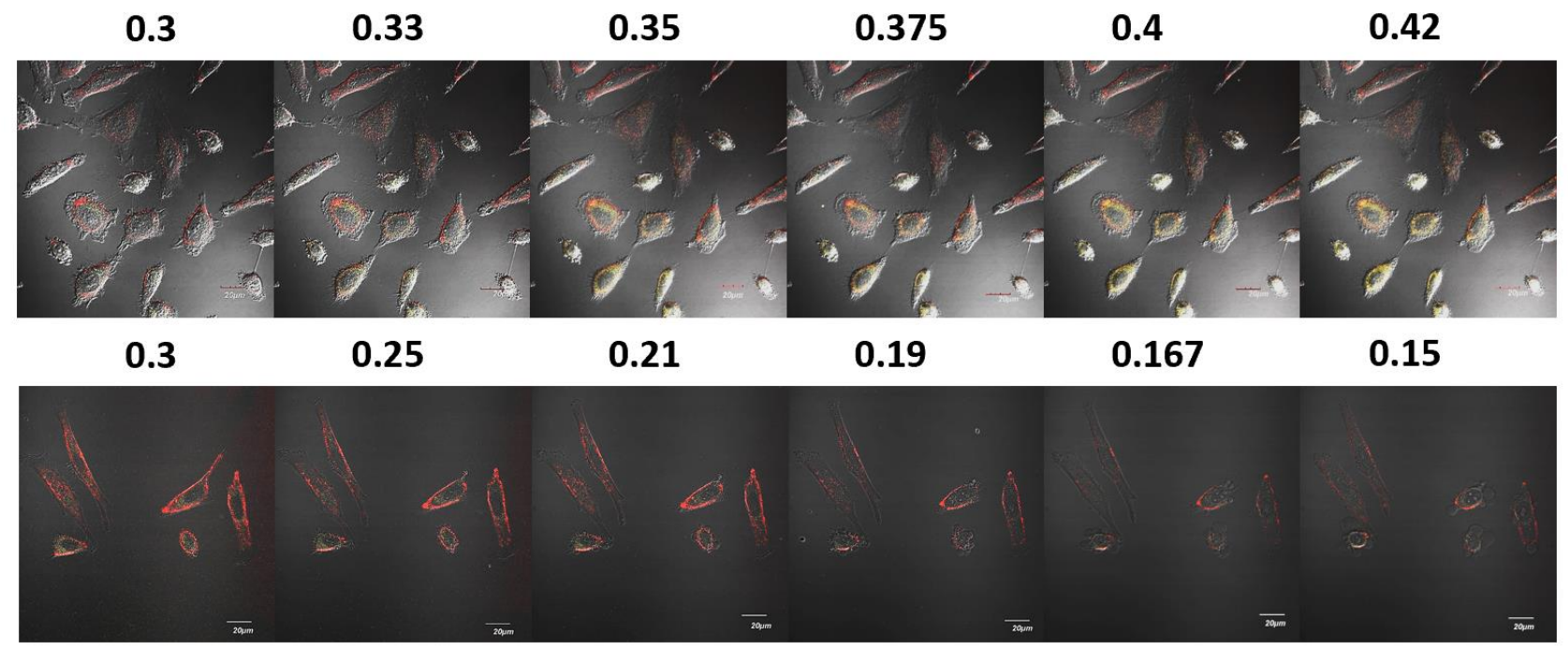

Figure S9 Merged channel with bright field of confocal images of live HeLa cells stained by TPNPDAC15 under hyper- and hypoosmotic pressure. Scale bars are $20 \mu \mathrm{m}$. 

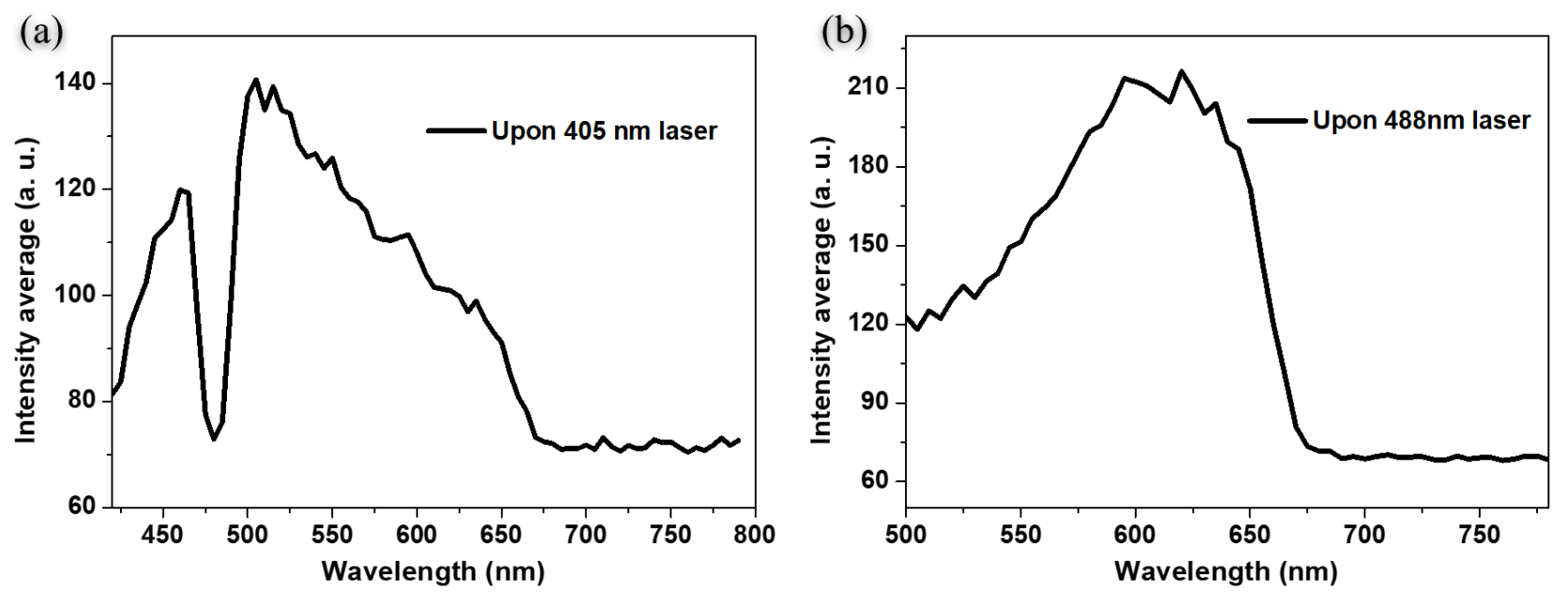

Figure S10 Plot of the mean emission intensity of HeLa cells stained by TPNPDA-C15 under $\Pi=0.4$ Osm from scans over various $\lambda$ zones from 400 to $800 \mathrm{~nm}$ with $5 \mathrm{~nm}$ integration. (a) Excited by $405 \mathrm{~nm}$ laser. (b) Excited by $488 \mathrm{~nm}$ laser. The plot was gained by a confocal microscope under lambda mode.
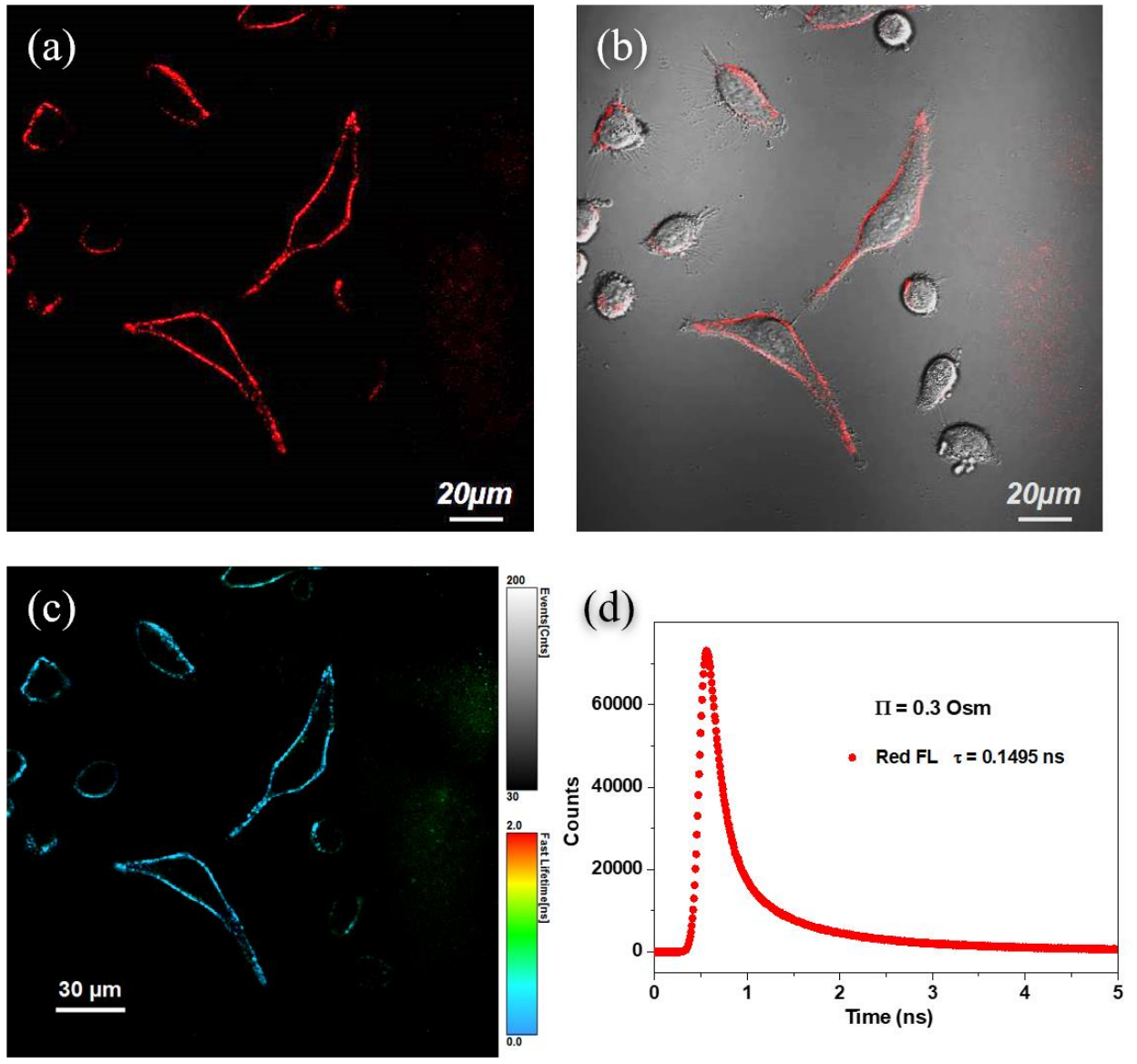

Figure S11 Confocal fluorescence images of HeLa cells stained with TPNPDA-C15 under $\Pi=0.3$ Osm 
in (a) red fluorescence channel, (b) merged channel with bright field. Scale bars are $20 \mu \mathrm{m}$. (c) Fluorescence lifetime images of HeLa cells stained with TPNPDA-C15 under $\Pi=0.3$ Osm in red channel. Fluorescence lifetime values from 0.0 to $2.0 \mathrm{~ns}$ are represented by pseudocolors from blue to red as shown in the color scale bar on the right. Scale bar is $30 \mu \mathrm{m}$. (d) Fluorescence decay curve and lifetime in red channels analyzed by FLIM system.
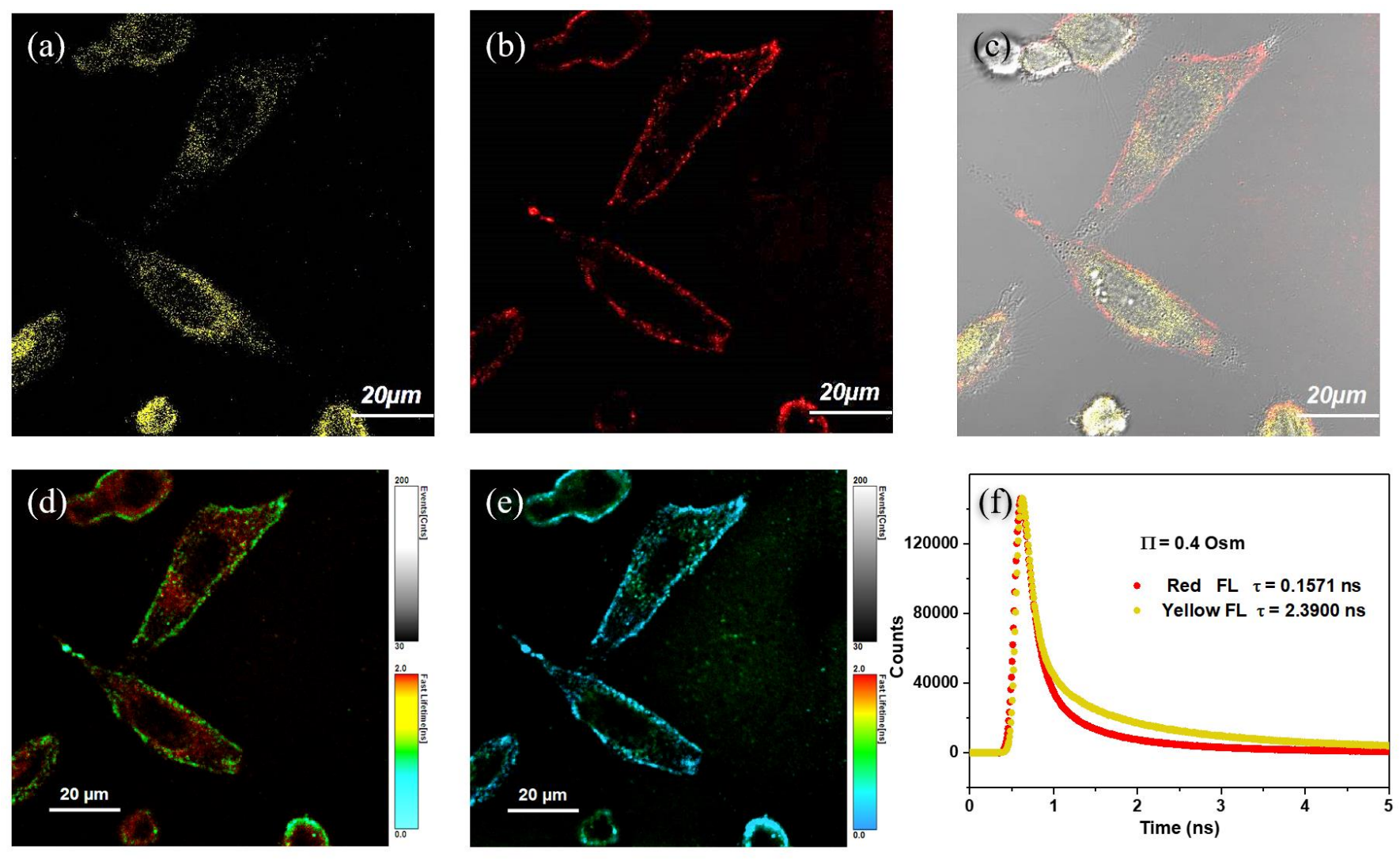

Figure S12 Confocal fluorescence images of HeLa cells stained with TPNPDA-C15 under $\Pi=0.4$ Osm in (a) short wavelength channel, (b) long wavelength channel, (c) merged channel with bright field. Fluorescence lifetime images of HeLa cells stained with TPNPDA-C15 under $\Pi=0.4$ Osm in (d) short wavelength channel and (e) long wavelength channel. Fluorescence lifetime values from 0.0 to $2.0 \mathrm{~ns}$ are represented by pseudocolors from blue to red as shown in the color scale bar on the right. Scale bars are $20 \mu \mathrm{m}$. (f) Fluorescence decay curves and lifetimes in yellow and red channels analyzed by FLIM system. 

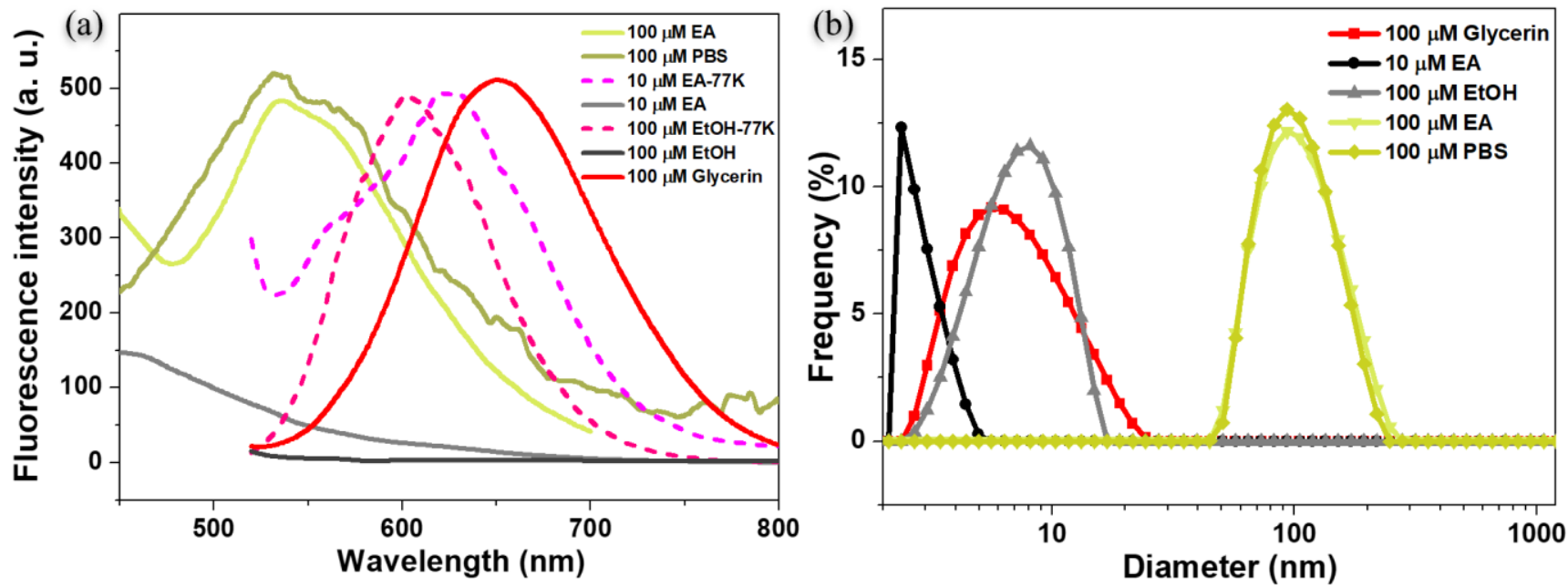

Figure S13 (a) Fluorescence spectra of TPNPDA-C15 in different solvents at $10 \mu \mathrm{M}$ or $100 \mu \mathrm{M}$ (dashed lines represent for fluorescence of samples at $77 \mathrm{~K}$ ). (b) Dynamic light scattering (DLS) analyses of TPNPDA-C15 in different solvents at $10 \mu \mathrm{M}$ or $100 \mu \mathrm{M}$.

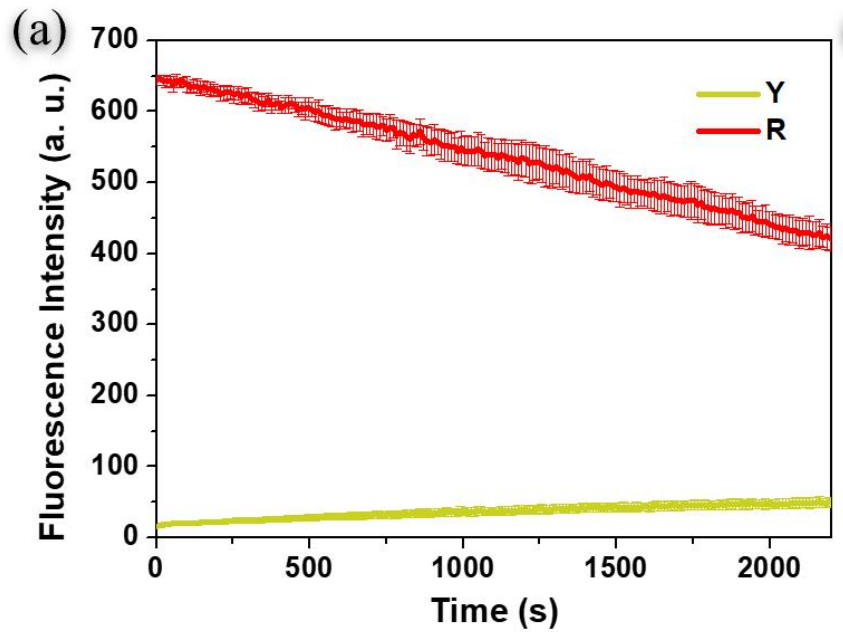

(b)
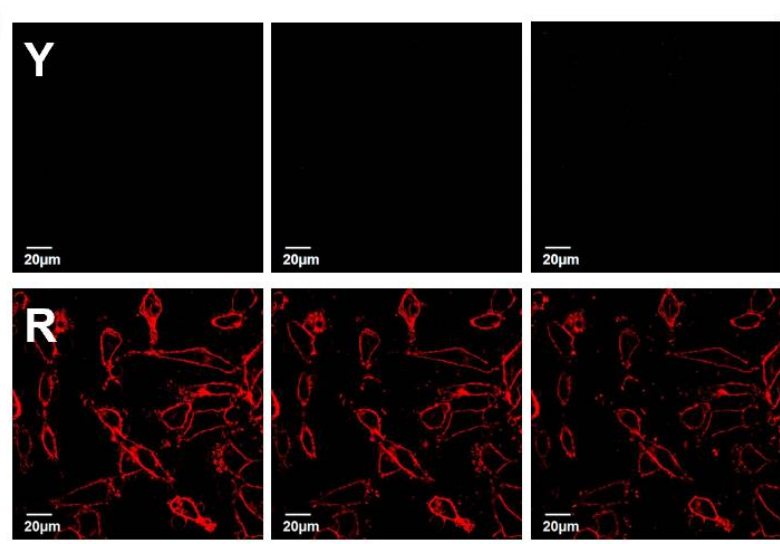

Os

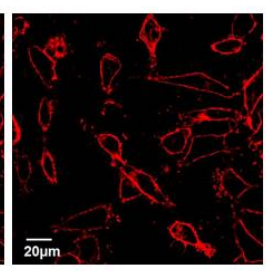

$1000 \mathrm{~s}$

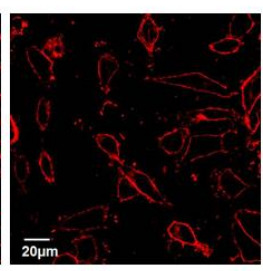

2000s

Figure S14 (a) Fluorescence intensity collected in long wavelength $(600-700 \mathrm{~nm})$ and short wavelength (440-540 nm) channel of HeLa cells stained by TPNPDA-C15 with increasing irradiation time of $405 \mathrm{~nm}$ and $488 \mathrm{~nm}$ laser, data collected through 800 continuous laser scans of CLSM. (b) Confocal fluorescence images of Hela cells stained by TPNPDA-C15 after irradiating for 0s, 1000s and 2000s (collected in short and long wavelength channel). Scale bars are $20 \mu \mathrm{m}$. 


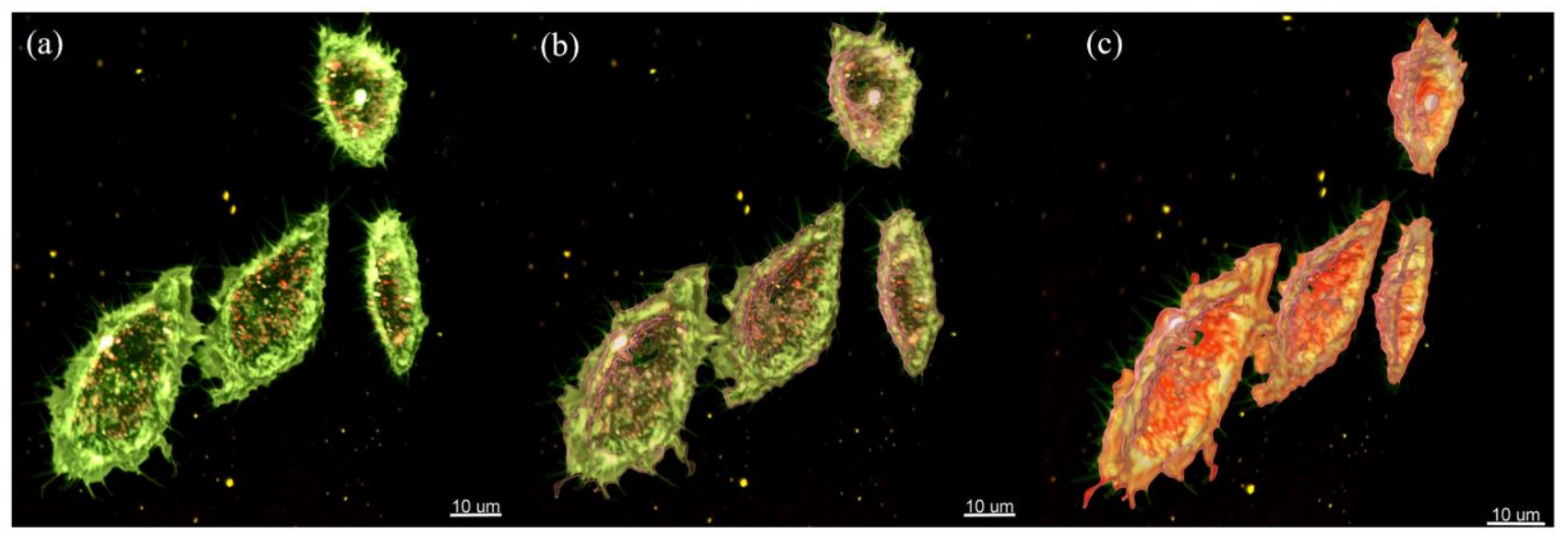

Figure S15 (a) Side view of 3D confocal fluorescence images of HeLa cells stained with TPNPDA-C15 and DiO (Merged channel). (b) Range of effective fluorescence signal in space of (a). (c) 3D surface built according to (b). Scale bars are $10 \mu \mathrm{m}$.
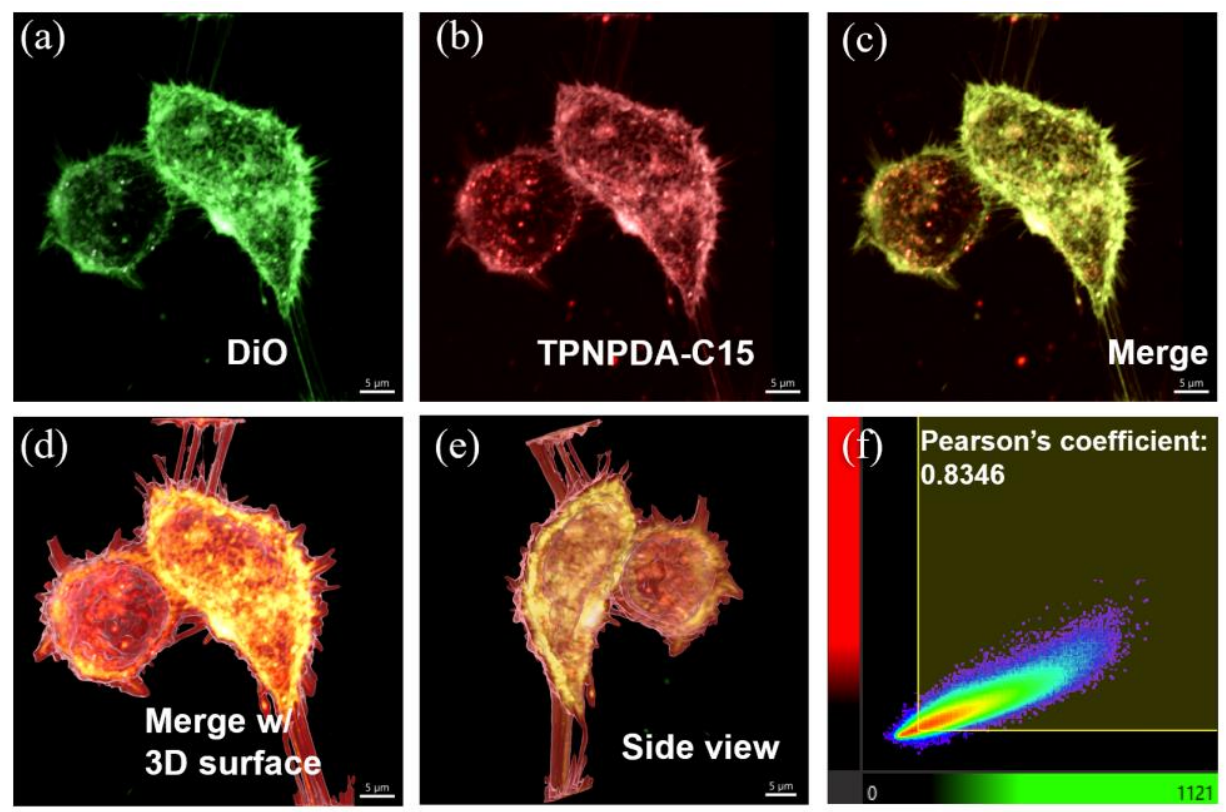

Figure S16 Three-dimensional imaging pictures of living HeLa cells co-stained with DiO and TPNPDAC15 at isosmotic pressure. (a) DiO Channel. (b) Long wavelength channel of TPNPDA-C15. (c) Merged channel. (d) Merged channel with 3D surface built according to effective fluorescence signal in space. (e) Side view of the 3D surface. Scale bars are $5 \mu \mathrm{m}$. (f) Co-localization scatter plot for long wavelength channel of TPNPDA-C15 with DiO in the established 3D surface. Pearson's correlation coefficient is 0.83. 

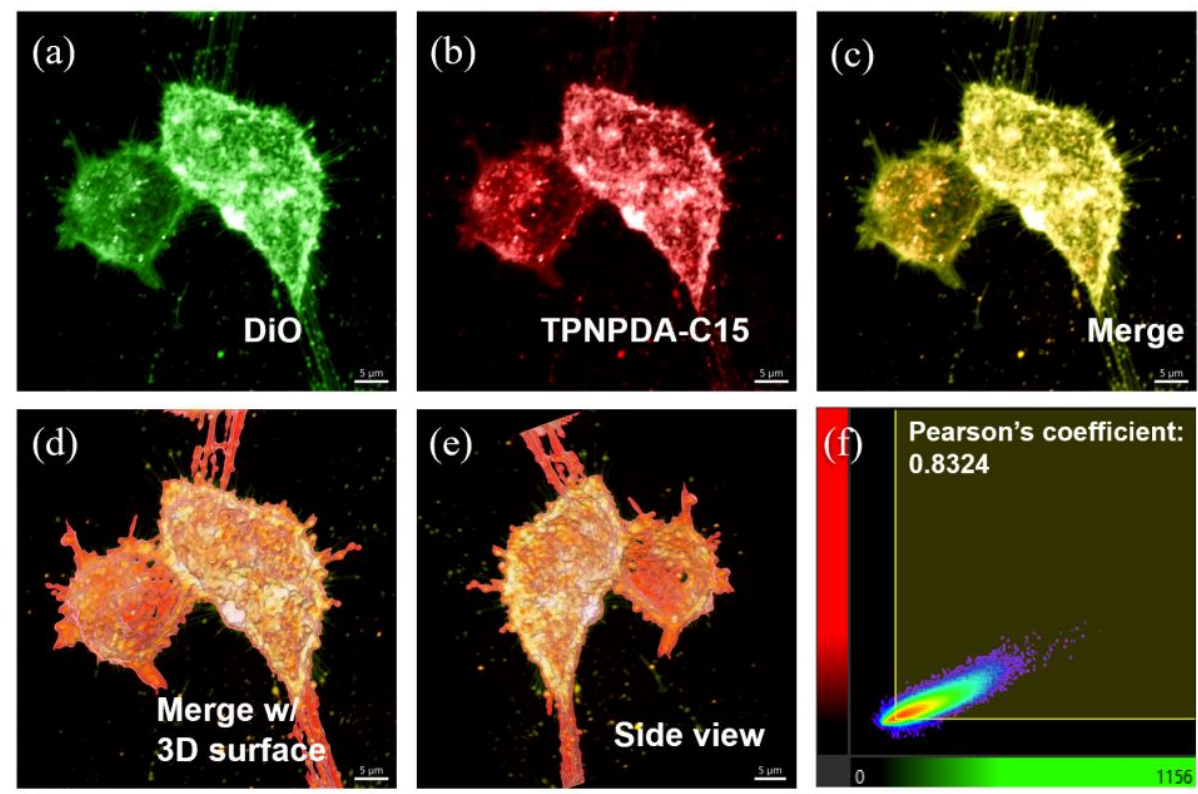

Figure S17 Three-dimensional imaging pictures of living HeLa cells co-stained with DiO and TPNPDAC15 at 0.35 Osm. (a) DiO Channel. (b) Long wavelength channel of TPNPDA-C15. (c) Merged channel. (d) Merged channel with 3D surface built according to effective fluorescence signal in space. (e) Side view of the $3 \mathrm{D}$ surface. Scale bars are $5 \mu \mathrm{m}$. (f) Co-localization scatter plot for long wavelength channel of TPNPDA-C15 with DiO in the established 3D surface. Pearson's correlation coefficient is 0.83.
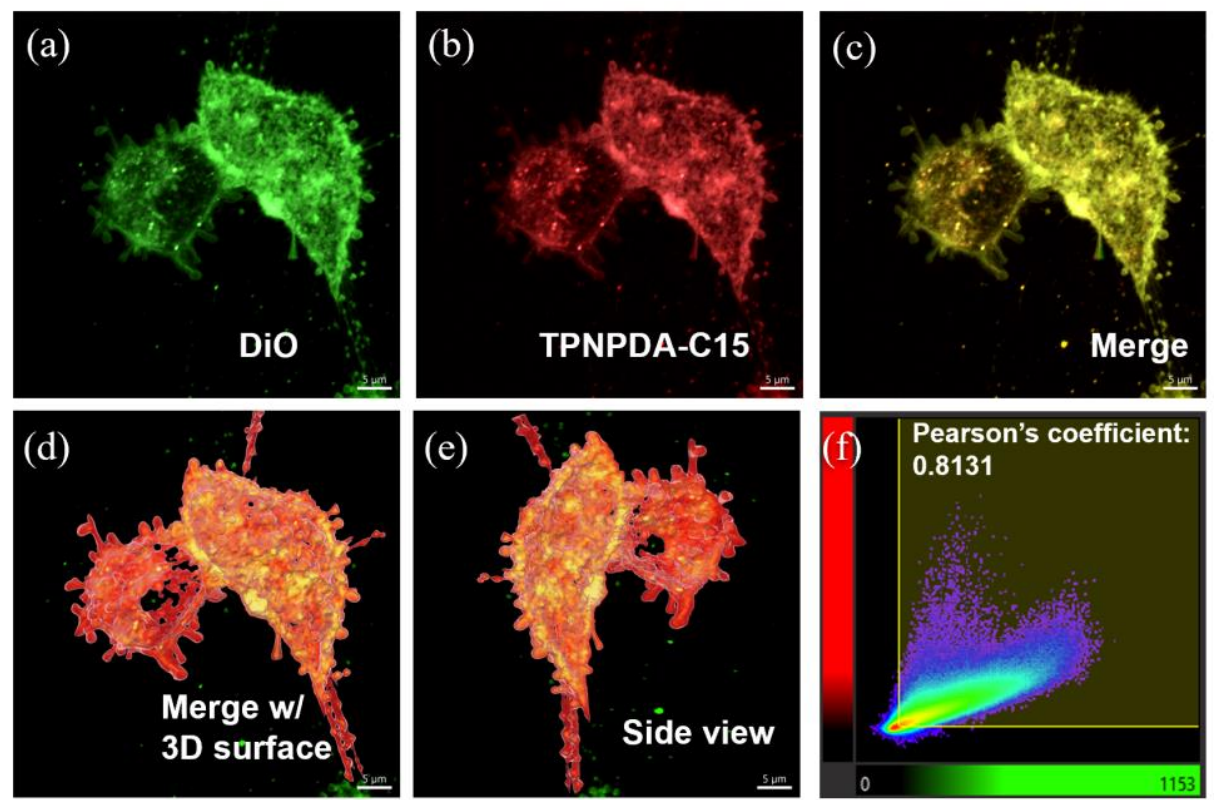

Figure S18 Three-dimensional imaging pictures of living HeLa cells co-stained with DiO and TPNPDAC15 at 0.4 Osm. (a) DiO Channel. (b) Long wavelength channel of TPNPDA-C15. (c) Merged channel. 
(d) Merged channel with 3D surface built according to effective fluorescence signal in space. (e) Side view of the 3D surface. Scale bars are $5 \mu \mathrm{m}$. (f) Co-localization scatter plot for long wavelength channel of TPNPDA-C15 with DiO in the established 3D surface. Pearson's correlation coefficient is 0.81.

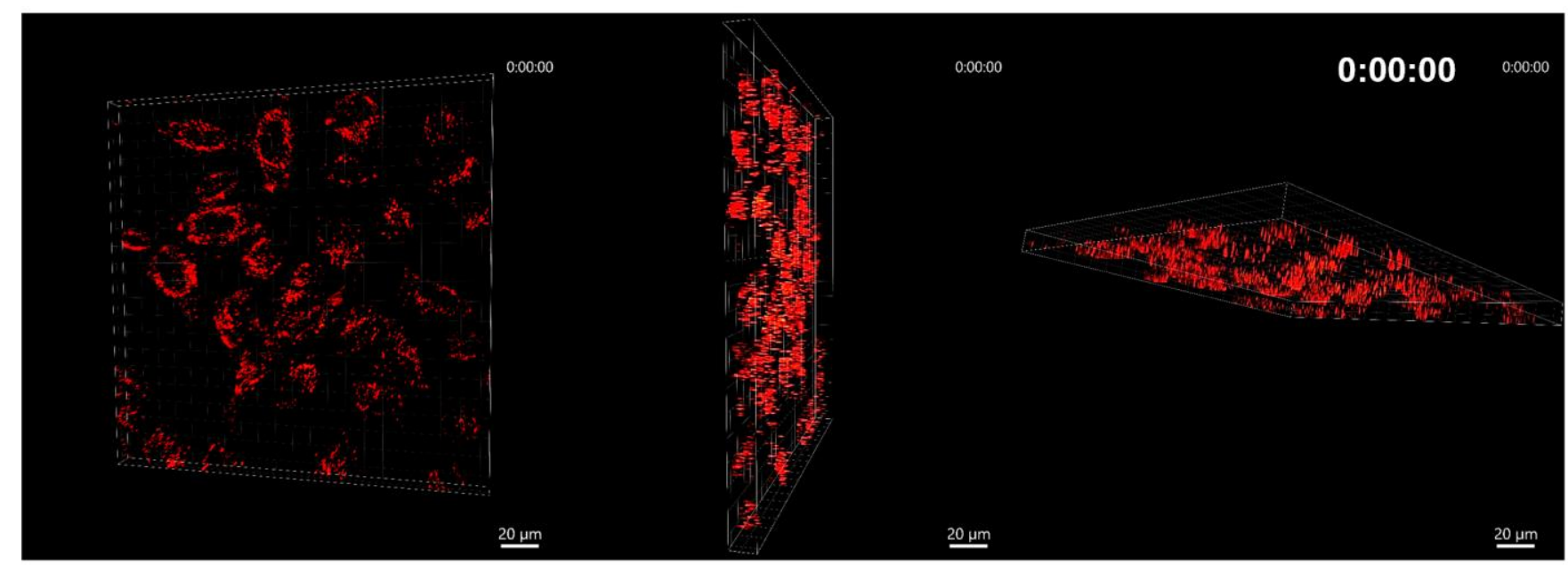

Figure S19 Front, side, and top view of 3D confocal fluorescence images of apoptotic HeLa cells stained with TPNPDA-C15 at $0 \mathrm{~h}$. Merged channel of short wavelength $(521 \pm 38 \mathrm{~nm})$ and long wavelength $(594 \pm 40 \mathrm{~nm})$ FL. Scale bars are $20 \mu \mathrm{m}$.

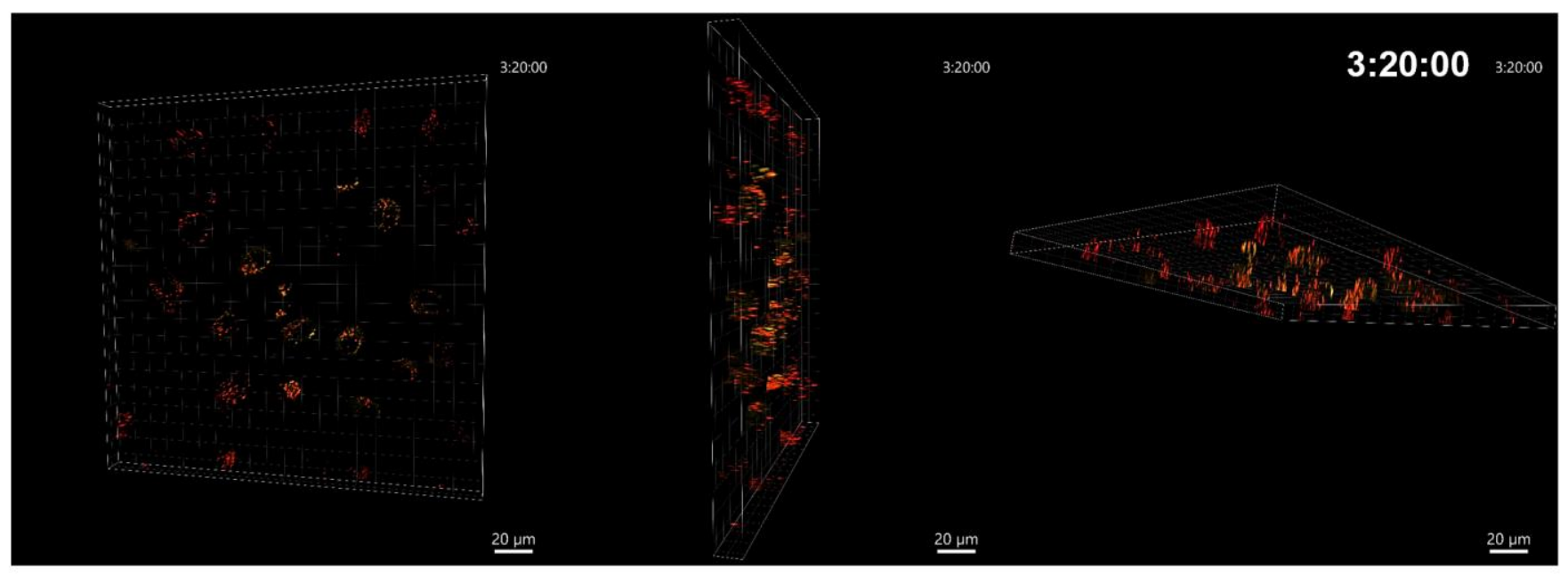

Figure S20 Front, side, and top view of 3D confocal fluorescence images of apoptotic HeLa cells stained with TPNPDA-C15 at 3 h 20 min. Merged channel of short wavelength $(521 \pm 38 \mathrm{~nm})$ and long wavelength $(594 \pm 40 \mathrm{~nm})$ FL. Scale bars are $20 \mu \mathrm{m}$. 


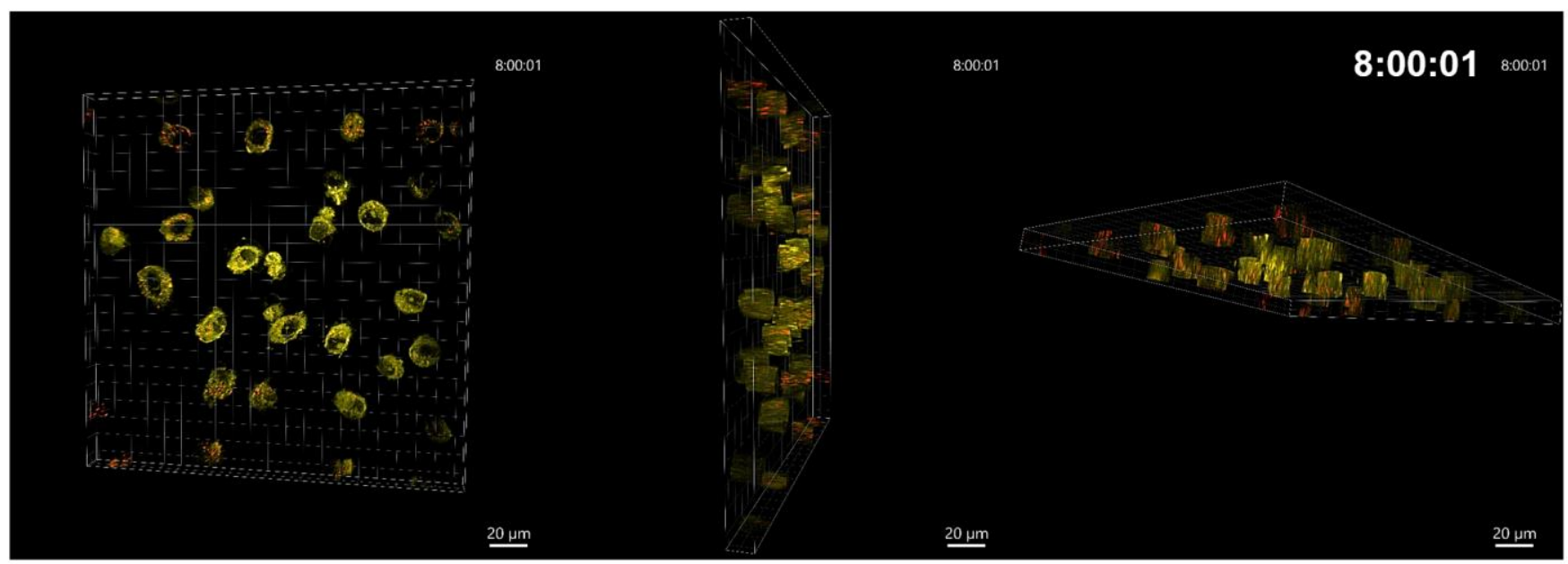

Figure S21 Front, side, and top view of 3D confocal fluorescence images of apoptotic HeLa cells stained with TPNPDA-C15 at $8 \mathrm{~h}$. Merged channel of short wavelength $(521 \pm 38 \mathrm{~nm})$ and long wavelength $(594 \pm 40 \mathrm{~nm})$ FL. Scale bars are $20 \mu \mathrm{m}$.

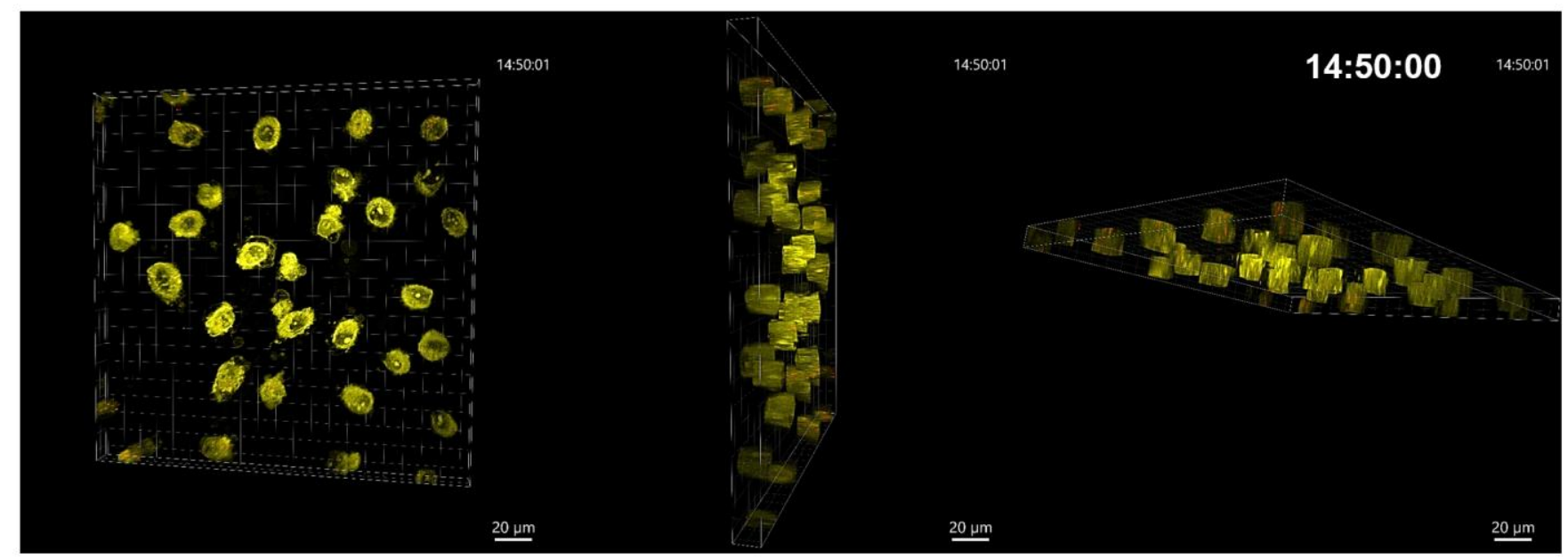

Figure S22 Front, side, and top view of 3D confocal fluorescence images of apoptotic HeLa cells stained with TPNPDA-C15 at $3 \mathrm{~h} 20$ min. Merged channel of short wavelength $(521 \pm 38 \mathrm{~nm})$ and long wavelength $(594 \pm 40 \mathrm{~nm})$ FL. Scale bars are $20 \mu \mathrm{m}$. 


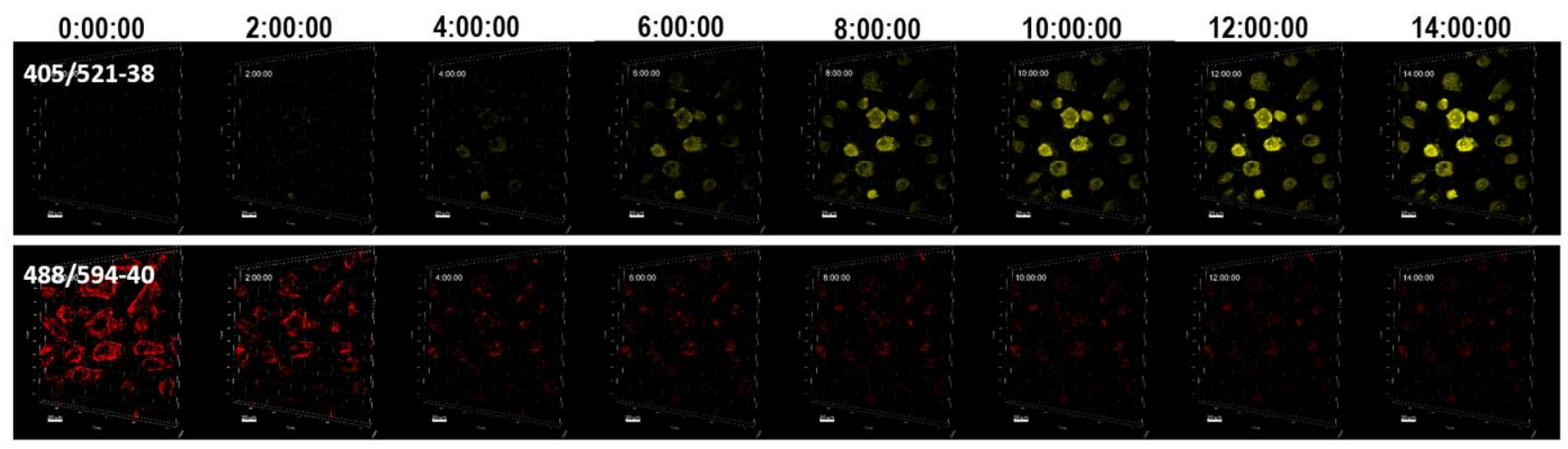

Figure S23 3D confocal fluorescence images of apoptotic HeLa cells stained with TPNPDA-C15 at different time (merged channel of yellow and red fluorescence). Scale bars are $20 \mu \mathrm{m}$.
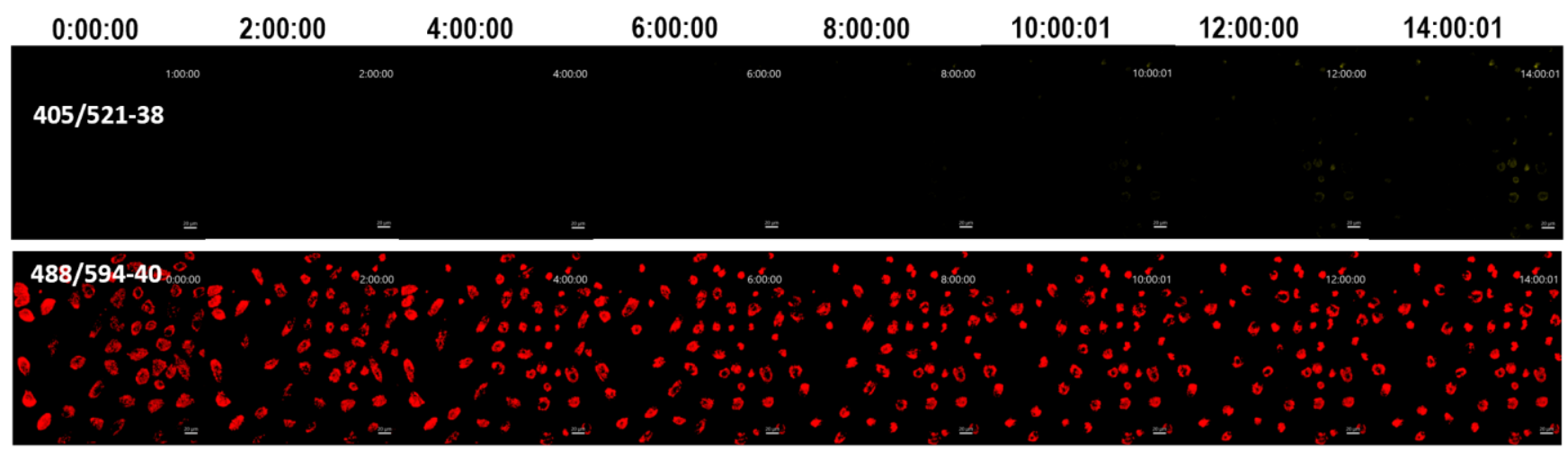

Figure S24 3D confocal fluorescence images of ferroptotic HeLa cells stained with TPNPDA-C15 at different time (merged channel of yellow and red fluorescence). Scale bars are $20 \mu \mathrm{m}$.

\section{Movies List}

Movie S1 3D co-localization of TPNPDA-C15 and DiO of Hela cells.

Movie S2 TPNPDA-C15 stained apoptotic HeLa cells recording for $15 \mathrm{~h}$ (Merged channel of yellow and red FL).

Movie S3 TPNPDA-C15 stained apoptotic HeLa cells recording for $15 \mathrm{~h}$ (Merged channel of yellow and red FL, with 3D spinning).

Movie S4 TPNPDA-C15 stained HeLa cells after the addition of $10 \mu \mathrm{M}$ erastin recording for $15 \mathrm{~h}$ (Merged channel of yellow and red FL). 
Movie S5 TPNPDA-C15 stained HeLa cells undergoing mitosis recording for $16 \mathrm{~h}$ (Merged channel of FL and bright field).

Movie S6 TPNPDA-C15 stained HeLa cells undergoing mitosis recording for $16 \mathrm{~h}$ (Merged channel of yellow and red FL).

\section{References}

1. Zheng, Y.; Ding, Y.; Ren, J.; Xiang, Y.; Shuai, Z.; Tong, A., Simultaneously and Selectively Imaging a Cytoplasm Membrane and Mitochondria Using a Dual-Colored Aggregation-Induced Emission Probe. Anal. Chem. 2020, 92 (21), 1449414500 . 